\title{
Atrial fibrillation inducibility after pulmonary vein isolation under general anaesthesia
}

\author{
Tomas Skala ${ }^{\mathrm{a}}$, Zbynek Tudos ${ }^{\mathrm{b}}$, Ondrej Moravec ${ }^{\mathrm{a}}$, Martin Hutyra ${ }^{\mathrm{a}}$, Jan Precek', Jitka Skalovac, Olga Klementovac, \\ Jana Zapletalova ${ }^{\mathrm{d}}$, Milos Taborskya
}

\begin{abstract}
Aims. Atrial fibrillation (AF) inducibility with rapid atrial pacing following AF ablation is associated with higher risk of AF recurrence. The predictive value of AF inducibility in paroxysmal AF patients after pulmonary vein isolation (PVI), done under general anaesthesia $(\mathrm{GA})$, remains questionable since GA might alter AF inducibility and/or sustainability. Methods. Consecutive patients $(n=120)$ with paroxysmal AF without prior catheter ablation $(C A)$ were enlisted in the study. All patients were ablated under GA. We have used a point-by-point CA and elimination of dormant conduction after adenosine in all patients. A predefined stimulation protocol was used to induce arrhythmias after PVI. Regular supraventricular tachycardias were mapped and ablated. Patients were divided into 3 subgroups - noninducible, inducible AF with spontaneous termination in five minutes, inducible AF without spontaneous termination. During 12 months of follow-up, all patients were examined four-times with 7-day ECG recordings.

Results. There was no statistical difference between the three subgroups in a rate of arrhythmia recurrence (11.1 vs. 27.5 vs. $27.3 \%, P=0.387$ ), despite a clear trend to a better success rate in the non-inducible group. The subgroups did not differ in left atrial (LA) diameter $(41.0 \pm 6,43.0 \pm 7,42.0 \pm 5 \mathrm{~mm}, P=0.962)$ or in any other baseline parameter.

Conclusion. AF inducibility as well as presence or absence of its early spontaneous termination after PVI done under general anaesthesia in paroxysmal AF patients were not useful as predictors of procedural failure.
\end{abstract}

Key words: atrial fibrillation, pulmonary veins isolation, inducibility, general anaesthesia

Received: December 3, 2018; Revised: February 8, 2019; Accepted: February 25, 2019; Available online: March 4, 2019 https://doi.org/10.5507/bp.2019.004

(c) 2020 The Authors; https://creativecommons.org/licenses/by/4.0/

${ }^{a}$ Department of Internal Medicine I - Cardiology, University Hospital Olomouc, Czech Republic

${ }^{b}$ Department of Radiology, University Hospital Olomouc, Czech Republic

'Department of Anesthesiology and Intensive Care Medicine, University Hospital Olomouc, Czech Republic

${ }^{d}$ Department of Biophysics, Faculty of Medicine and Dentistry, Palacky University Olomouc, Czech Republic

Corresponding author:Zbynek Tudos, e-mail: zbynek.tudos@seznam.cz

\section{INTRODUCTION}

The absence of atrial fibrillation (AF) inducibility with rapid atrial pacing following $\mathrm{AF}$ ablation is associated with a lower risk of AF recurrence ${ }^{1}$. AF inducibility in paroxysmal $\mathrm{AF}$ patients has been used to guide further left atrial substrate modification ${ }^{2,3}$. In persistent and longstanding persistent $\mathrm{AF}$, the generally accepted end-point is non-inducibility of any atrial tachyarrhythmia, since inducibility of any atrial tachyarrhythmia after ablation in these patients is linked to poor prognosis ${ }^{4}$. In paroxysmal $\mathrm{AF}$, pulmonary vein isolation (PVI) is still the end-point of ablation. Despite recent technological improvements in PVI, single catheter ablation (CA) of paroxysmal AF consisting just of PVI, is rarely reported to have much higher success rate than $70 \%$ (ref. . $^{5}$. It is crucial to identify that one third of patients in whom PVI will fail. Apart from left atrial (LA) diameter, no other procedural failure predictors are widely accepted ${ }^{6}$. As mentioned above, AF inducibility after PVI is considered to be linked to poor prognosis. However, there is a significant heterogeneity between studies in the sites of stimulation, method of stimulation, use of pharmacological provocation, and in the definition of inducibility based on AF duration? ${ }^{7}$. Thus, the predictive value of $\mathrm{AF}$ inducibility in paroxysmal $\mathrm{AF}$ patients after PVI remains questionable. Moreover, only limited data are available about AF inducibility after PVI under general anaesthesia (GA). The autonomic nervous system exerts important effects upon AF initiation. The strategy of anaesthesia used during AF ablation may impact the provocation of AF triggers. AF inducibility and presence of extra-pulmonary (nonPV) foci may be suppressed in GA $\left(\right.$ ref. $\left.^{8-10}\right)$.

\section{METHODS}

\section{Patient group}

A total of 120 patients indicated for catheter ablation of paroxysmal atrial fibrillation were enlisted in the study. Patients were classified as having paroxysmal AF according to current guidelines ${ }^{5}$.

Inclusion criteria were paroxysmal $\mathrm{AF}$, at least 3 episodes of AF in the last 6 months, age $>18$ years, sinus rhythm at the beginning of the ablation and a signed informed consent for the study.

Exclusion criteria were persistent or long-standing persistent AF, structural heart disease, moderate-to-severe or 
severe valve disease or a history of valve disease surgery, left ventricular ejection fraction $<35 \%$, chronic use of amiodarone, presence of intra-cardiac thrombi as documented by transesophageal echocardiography, uncontrolled thyroid disorders, pregnancy, breast feeding, severe renal dysfunction, inadequate follow-up and inability to provide informed consent.

\section{Procedural details}

\section{Procedures before catheter ablation}

All patients underwent transesophageal and transthoracic echocardiography within $24 \mathrm{~h}$ before ablation and cardiac computed tomography focused on pulmonary veins (PVs) anatomy within 7 days before ablation.

On transthoracic echocardiography, LA diameter was measured in the parasternal long axis, perpendicularly to the LA walls. LA diameter was measured in end-systole, from leading edge of the posterior aortic wall to the leading edge of the posterior LA wall.

At least 3 days before ablation, antiarrhythmic medication was discontinued in all patients. No patient was on amiodarone during 3 months before ablation. Written informed consent was obtained from all subjects.

\section{Catheter ablation}

Catheter ablation was done under GA in all patients. Diprivan (Propofol), Sufentanil citrate (Sufentanil), Rocuronium bromide (Esmeron) and Sevoflurane (Sevorane) were used in GA. Two sheaths were introduced via the femoral vein for intra-cardiac echocardiography probe (AcuNav ultrasound catheter, Siemens Healthineers, USA) and decapolar diagnostic coronary sinus catheter (Inquiry ${ }^{\mathrm{TM}}$, St. Jude Medical). Two steerable transseptal sheaths (Agilis ${ }^{\mathrm{TM}}$ NxT Steerable Introducer, St. Jude Medical) were introduced via the femoral vein. A double transseptal puncture was performed in all patients. After transseptal puncture a 3D electro-anatomical map was performed using Carto ${ }^{\mathrm{TM}}$ (Biosense Webster). A fast anatomical map was created consistently in all patients. The anatomical map was merged with a 3D model of LA using CartoMerge ${ }^{\mathrm{TM}}$ technology. A point-by-point radiofrequency wide-antral ablation (Fig. 1) was done in all patients. Ablation energy was set to $25-30 \mathrm{~W}$ in all patients with cool flow of $20 \mathrm{~mL} / \mathrm{min}$. Lasso ${ }^{\mathrm{TM}}$ catheter (Biosense Webster) was used to validate PVI (entry block) in all patients. After PVI we waited for $20 \mathrm{~min}$ and then adenosine was applied intravenously to test dormant conduction (DC) using a double lasso technique. At least 18 $\mathrm{mg}$ of adenosine was used for the test. The dose was considered sufficient if it resulted in second or third degree atrioventricular block. Additional ablation was performed in case of PVs with conduction recovery after adenosine testing. To determine whether DC was eliminated after re-ablation, another adenosine testing was performed.

After PVI and a negative adenosine test, a predefined stimulation protocol was used to induce any type of supraventricular arrhythmia (SVT). Just before the start of this stimulation protocol, we checked for PVI again.

\section{Stimulation protocol}

All patients underwent a predefined stimulation protocol from a single site (proximal coronary sinus). Pacing output was $20 \mathrm{~mA}$, pulse width $1.0 \mathrm{~ms}$. The protocol consisted of 4 parts.

1) Atrial burst pacing (15-beat drive train) with a cycle length $300 \mathrm{~ms}$.

2) Atrial burst pacing (15-beat drive train) with a cycle length $250 \mathrm{~ms}$.

3) Atrial burst pacing (15-beat drive train) with a cycle length $200 \mathrm{~ms}$.

4) Auto-decremental (RAMP) pacing from 300 to $200 \mathrm{~ms}$. Pacing at each decrement $(-10 \mathrm{~ms})$ lasted for $3 \mathrm{~s}$, except the last one (200 ms) which lasted for $5 \mathrm{~s}$.

\section{Ablation of arrhythmias other than AF}

If $\mathrm{AF}$ was induced, the stimulation protocol was ended (see AF induction).

If an arrhythmia other than AF was induced during the initial stimulation protocol, these arrhythmias were to be mapped and ablated:

1) Frequent $(\geq 2 / \mathrm{min})$ supraventricular extrasystoles (SVES)

2) Regular sustained atrial tachycardia (AT)

3) Typical atrial flutter

4) Atrioventricular nodal re-entry tachycardia (AVNRT)

5) Atrioventricular re-entry tachycardia (AVRT) with a concealed accessory pathway (AP).

In case of a supraventricular tachycardia suspected of AVNRT/AVRT a complex electrophysiological evaluation was performed to evaluate duality of atrioventricular nodal conduction and presence of AP.

Cavotricuspid isthmus (CTI) was ablated even in case of a short non-sustained regular atrial tachycardia with proximal to distal coronary sinus activation.

After these arrhythmias were ablated, the stimulation protocol was performed again. Once again, just before the start of this stimulation protocol, we checked for PVI once more. The main focus at this second stimulation was set on AF. A sustained SVT could have been ablated, but a non-sustained one could not.

\section{AF induction}

If AF was induced, we waited for $5 \mathrm{~min}$, since induced AF lasting for a shorter time could be non-specific. This interval was based on previous definitions ${ }^{7,11}$. If AF did not spontaneously terminate during this time, a cardioversion was performed and the procedure was ended.

\section{Follow-up}

Four 7-day ambulatory ECG recordings were scheduled every 3 months (3, 6, 9, 12 months after ablation) with a subsequent thorough clinical examination. The patients were instructed about the need for an early examination in the out-patient department in case of palpitations with an effort to document a possible arrhythmia in the period of time between 7-day ambulatory ECG monitoring. Any documented arrhythmia (atrial flutter, AT, AF) 
lasting more than $30 \mathrm{~s}$ until the end of the follow-up was considered an arrhythmia recurrence. A blanking period was not used. 12 months after ablation, all patients were divided according to presence or absence of arrhythmia recurrence into two groups (A-AF-free, B-AF-recurrence).

\section{Statistical evaluation}

The statistical software IBM SPSS Statistics ver. 22 was used to perform data analysis. Subgroups of patients were compared for quantitative variables using Student's t-test or Mann-Whitney U-test depending on data normality. For qualitative parameters, the subgroups of patients were compared using chi-square test or Fisher's exact test. Data normality was verified using Shapiro-Wilk test. All tests were done at a level of significance 0.05 .

\section{RESULTS}

\section{AF inducibility}

AF was not inducible in $18 / 120$ patients. AF was inducible in 102/120 patients. It spontaneously terminated in 91/102 patients with inducible AF. AF lasted for $>5$ min and had to be terminated in 11/102 patients with inducible AF.

\section{Follow-up}

All 120 patients completed a 1-year long follow-up. No patient was lost during the follow-up. PVI (entry and exit block) was achieved in all patients. No major complications such as cardiac tamponade, stroke, atrio-esophageal fistula and/or PV stenosis were documented in the study sample. 2 patients presented with a large groin hematoma prolonging the hospitalization for 2 and 3 days, respectively. Both were treated conservatively without consequences. All patients had four 7-day ambulatory ECG recordings every 3 months $(3,6,9,12$ months after ablation) with a subsequent thorough clinical examination in all patients.

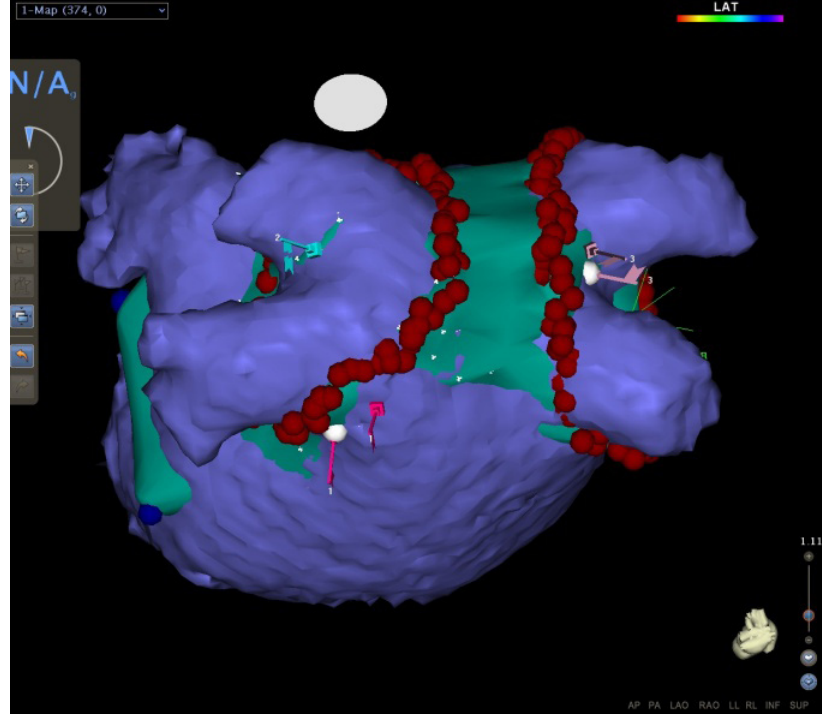

Fig. 1. CARTO3 anatomical model of left atrium. Wide encircling lesions around ostia of ipsilateral pulmonary veins.

Red dots $=$ ablated areas.

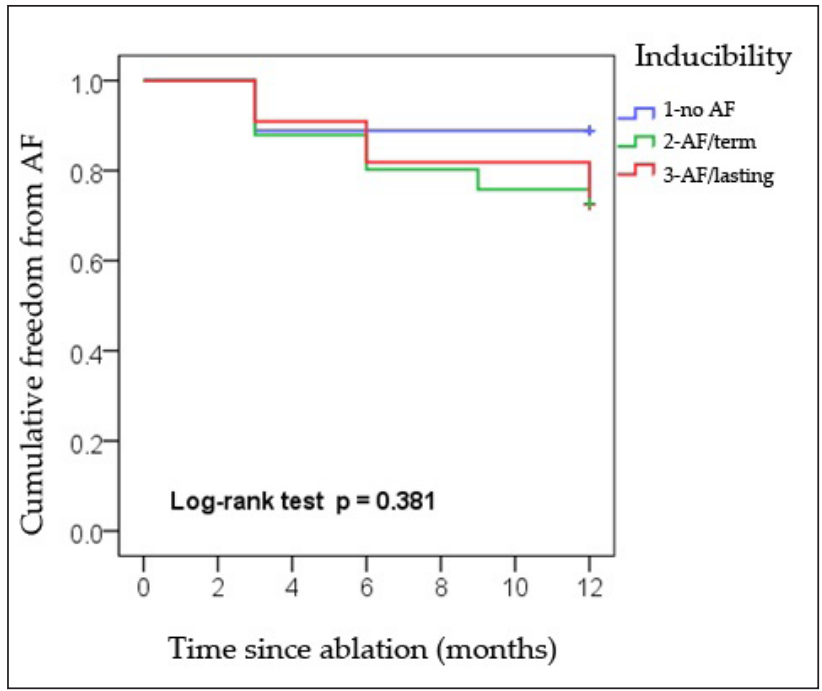

Fig. 2. Kaplan-Meier analysis of arrhythmia-free survival rate 0-12 months after ablation.

Table 1. Arrhythmias inducible in individual subgroups.

\begin{tabular}{lccc}
\hline Arrhythmia induction & $\begin{array}{c}\text { AF non-inducible } \\
(\mathrm{n}=18)(\%)\end{array}$ & $\begin{array}{c}\text { AF termination } \\
(\mathrm{n}=91)(\%)\end{array}$ & AF cardioversion $(\mathrm{n}=11)(\%)$ \\
\hline CTI & $7(38.8)$ & $35(38.5)$ & $3(27.3)$ \\
SVES/AT nonPV - focal & $1(5.6)$ & $6(6.6)$ & $0(0)$ \\
SVES posterior wall LA (box) & $0(0)$ & $1(1.1)$ & $0(0)$ \\
AVRT (concealed AP) & $0(0)$ & $2(2.2)$ & $0(0)$ \\
AVNRT & $2(11.1)$ & $3(3.3)$ & $0(0)$ \\
\hline
\end{tabular}

Table 2. AF Inducibility impact on arrhythmia recurrence 12 months after ablation.

\begin{tabular}{|c|c|c|c|c|c|c|c|}
\hline & \multicolumn{6}{|c|}{ Inducibility } & \multirow{2}{*}{$P$} \\
\hline & & AF non-inducible $(\mathrm{n}=18)$ & & AF termination $(n=91)$ & \multicolumn{2}{|c|}{ AF cardioversion $(n=11$} & \\
\hline AF recurrence - & 16 & $88.9 \%$ & 66 & $72.5 \%$ & 8 & $72.7 \%$ & \multirow{2}{*}{0.387} \\
\hline AF recurrence + & 2 & $11.1 \%$ & 25 & $27.5 \%$ & 3 & $27.3 \%$ & \\
\hline
\end{tabular}


Table 3. Arrhythmia-free survival rate $0-12$ months after ablation.

\begin{tabular}{lcccc}
\hline & & \multicolumn{3}{c}{ Mean $^{\mathrm{a}}$} \\
\cline { 2 - 5 } Inducibility & Estimate & Std. Error & \multicolumn{2}{c}{$95 \%$ Confidence Interval } \\
None & & & Lower Bound & Upper Bound \\
AF/ termination & 11.0 & 0.7 & 9.7 & 12.3 \\
AF/cardioversion & 10.3 & 1.1 & 8.7 & 11.0 \\
Overall & 10.6 & 0.3 & 9.9 & 11.0 \\
\hline
\end{tabular}

Means and Medians for Survival Time.

${ }^{a}$ Estimation is limited to the largest survival time if it is censored.

Table 4. Test of equality of survival distributions for the different levels of patient subgroups according to inducibility.

\begin{tabular}{cccc}
\hline & Chi-Square & df & Sig. \\
\hline Log Rank (Mantel-Cox) & 1.928 & 2 & 0.381 \\
\hline
\end{tabular}

Table 5. The difference in LA diameter in different inducibility groups.

\begin{tabular}{ccccc}
\hline Inducibility & None $(\mathrm{n}=18)$ & $\mathrm{AF} /$ term $(\mathrm{n}=91)$ & $\mathrm{AF} /$ version $(\mathrm{n}=11)$ & $P$ \\
\hline LA $(\mathrm{mm})$ & $41.0 \pm 6$ & $43.0 \pm 7$ & $42.0 \pm 5$ & 0.962 \\
\hline
\end{tabular}

LA measured in parasternal long-axis on transthoracic echocardiography, Students' t-test.

Table 6. The difference in baseline clinical parameters before ablation in patients according to inducibility.

\begin{tabular}{lcccc}
\hline & None $(\mathrm{n}=18)$ & $\begin{array}{c}\text { Inducibility } \\
\text { AF/termination }(\mathrm{n}=91)\end{array}$ & $\begin{array}{c}\text { AF/cardioversion }(\mathrm{n}=11) \\
(\%)\end{array}$ & $P$ \\
\hline Arterial hypertension & $11(61.1)$ & $55(60.4)$ & $8(72.7)$ & 0.767 \\
Diabetes mellitus & $3(16.7)$ & $7(7.7)$ & $0(0.0)$ & 0.361 \\
Heart failure & $1(5.6)$ & $5(5.5)$ & $1(9.1)$ & 0.798 \\
Coronary artery disease & $0(0.0)$ & $6(6.6)$ & $0(0.0)$ & 0.771 \\
Myocardial infarction & $0(0.0)$ & $4(4.4)$ & $0(0.0)$ & 1 \\
Hyperlipidaemia & $6(33.3)$ & $33(36.3)$ & $4(36.4)$ & 1 \\
Stroke & $1(5.6)$ & $5(5.5)$ & $0(0.0)$ & 1 \\
Obstructive sleep apnoea & $1(5.6)$ & $3(3.3)$ & $1(9.1)$ & 0.349 \\
\hline
\end{tabular}

\section{Dormant conduction}

In group-1 (AF-non-inducible), DC was present in $2 / 18(11.1 \%)$. In group-2 (AF/termination), DC was present in 29/91 (31.9\%). In group-3 (AF/cardioversion), DC was present in $1 / 11(9.1 \%)$. DC was eliminated in all patients before arrhythmia induction.

\section{Inducible arrhythmias other than AF}

Arrhythmias inducible in individual subgroups are listed in Table 1

\section{Freedom from AF 12 months after ablation}

There was no statistical difference between the three subgroups ( 11.1 vs. 27.5 vs. $27.3 \%, P=0.387)$ in freedom from AF 12 months after ablation (Table 2, 3, 4), despite a clear trend to a better success rate in the non-inducible group (Fig. 2).

The subgroups did not differ in left atrial (LA) diameter $(41.0 \pm 6,43.0 \pm 7,42.0 \pm 5, P=0.962)$ or in any other baseline parameter (Table 5 ).
There were no significant differences in baseline clinical parameters before ablation in patients according to inducibility (Table 6).

\section{DISCUSSION}

Despite advances in new technologies, one third of patients afflicted with paroxysmal AF who were treated with PVI have a documented AF recurrence. A possible PV reconduction cannot be held responsible for every AF recurrence. A number of patients have AF recurrence despite the evidence of all PVs isolated during a second procedure $^{12}$. A method for predicting procedural failure would be of a great clinical relevance. The role of arrhythmia inducibility in paroxysmal AF is still questionable. AF inducibility with a rapid atrial pacing following AF ablation is associated with a higher risk of AF recurrence and was used to guide further left atrial substrate modification ${ }^{1-3}$. However, the sensitivity and specificity of the 
AF inducibility test is difficult to estimate, since inducible and sustained AF is common in patients in the absence of structural heart disease or clinical AF (ref. ${ }^{7}$ ). Especially in patients under GA, it is not clear what it means if we can or cannot induce AF after PVI, and if AF terminates or not. AF inducibility and presence of extra-pulmonary (nonPV) foci may be suppressed in GA (ref. ${ }^{8,9}$ ). We can speculate that an inducible AF could be a sign of a larger substrate. So we could have eliminated a dominant location of AF triggers, but we have not treated enough substrate to sustain AF and we can anticipate a recurrence. On the other hand, it could mean that we have eliminated AF triggers and by the stimulation we just substitute the removed triggers and thus create AF iatrogenically. There was no statistically significant difference in our patient group between the subgroups with and without inducible AF. Although the trend towards a better outcome in the non-inducible subgroup was clearly evident, the stimulation result after PVI was not useful in predicting clinical outcome.

Our finding is in contrast to studies reporting that the absence of AF inducibility with rapid atrial pacing following AF ablation is associated with a lower risk of AF recurrence ${ }^{2-3}$. However, AF inducibility may be a nonspecific sign. According to Huang et al., AF can be induced by pacing in $26 \%$ of patients without a history of AF (ref. ${ }^{13}$ ). Santangeli et al. studied the inducibility of atrial arrhythmia or change in inducibility status after PVI and non-PV trigger ablation. Their main finding was that inducible and sustained $\mathrm{AF}$ ( $\geq 5 \mathrm{~min}$ ) is common in patients without structural heart disease or clinical AF (ref. ${ }^{14}$ ). Our results in patients under GA support their findings. We have shown that AF inducibility in paroxysmal AF is not associated with significantly worse long-term freedom from recurrent arrhythmia and should not be used as an ablation endpoint, or to support the appropriateness of additional ablation lesion sets.

It is also not clear, if the absence of spontaneous AF termination in inducible patients is a mark of greater structural atrial impairment. It might also be just a nonspecific sign. According to Kumar et al., a sustained AF (>5 min) is common (29.5\%) in patients in the absence of structural heart disease or clinical AF, and its incidence varies according to gender, method of induction, and number of inductions ${ }^{7}$. In our patient group, there was no difference at all between patients with spontaneous AF termination and those with AF lasting for more than 5 min leading to cardioversion. The presence or absence of spontaneous AF termination did not correlate with arrhythmia-free survival rate. Our data are in agreement with the above-mentioned finding by Santangeli et al. ${ }^{14}$. Our goal was to find out the clinical usefulness of inducibility of AF specifically. Inducibility of regular tachyarrhythmias is generally linked to a higher probability of their recurrence ${ }^{15}$. For that reason, we decided to eliminate all arrhythmias inducible after PVI. In case of AT suspected of typical flutter, we ablated CTI. In case of frequent SVES or inducible AT, we mapped and ablated those arrhythmias. If AVNRT or AVRT were suspected, we also tried to ablate them. All this was done to ensure "only" a non-organized AF was inducible during the second round of stimulation. A higher percentage of CTI ablation in our patient group compared to other trials was caused by our very low threshold for CTI ablation that is based on safety and simplicity of CTI ablation if intracardiac echocardiography is used ${ }^{16}$. In the AF/termination subgroup, DC after adenosine test was more frequent. However, in all cases a DC was eliminated by additional ablation before the stimulation protocol started.

\section{Study limitations}

Patients were not randomized to additional ablation based on their inducibility status. Our goal was, however, to find out if AF inducibility and/or sustainability after PVI done in GA is linked with a worse procedure outcome.

GA could have suppressed nonPV foci, so we might have missed them. This is nevertheless true for all subgroups alike and we specifically tried to discover the impact of inducibility in GA patients.

We did not use isoprenaline to enhance AF inducibility. The reason is that isoprenaline is not widely used in the first ablation of paroxysmal AF and we did not want to deflect from a common practice.

Different types of stimulation protocols can lead to different results. There is no "golden standard" for a stimulation protocol. It differs in some details from lab to lab and from study to study. However, the type of the stimulation protocol cannot have any impact on the duration (sustainability) of AF, once it is induced.

Despite an adequate waiting time, dose of adenosine and the fact that inducibility was tested after elimination of DC and additional PVI check, we cannot rule out that in some patients a PV reconduction might have occurred during the follow-up with unclear impact on arrhythmia recurrence.

\section{CONCLUSION}

AF inducibility as well as presence or absence of its early spontaneous termination after PVI done under general anaesthesia in paroxysmal AF patients were not useful predictors of procedural failure. In these patients AF inducibility as well as lack of its early spontaneous termination might only be nonspecific signs not predicting AF recurrence.

\section{ABBREVIATIONS}

AF, atrial fibrillation; AP, accessory pathway; AT, atrial tachycardia; AVNRT, atrioventricular nodal re-entry tachycardia; AVRT, atrioventricular re-entry tachycardia; CA, catheter ablation; CTI, cavotricuspid isthmus; DC, dormant conduction; GA, general anaesthesia; LA, left atrial; PV, pulmonary vein; PVI, pulmonary veins isolation; SVT, supraventricular arrhythmias; SVES, supraventricular extrasystoles. 
Author contributions: TS: study design, performing CA, complete patient FU, data analysis, data interpretation, manuscript writing and literature search; ZT: study design, acquiring $\mathrm{CT}$, data analysis, data interpretation, manuscript writing and literature search, critical revision of the manuscript; OM: performing CA, patient FU; MH, JP: data collection, data interpretation, critical revision of the manuscript; JS, OK: general anaesthesia, data collection, critical revision of the manuscript; JZ: statistical analysis; MT: critical revision of the manuscript, final approval of the manuscript.

Conflict of interest statement: None declared.

\section{REFERENCES}

1. Chang SL, Tai CT, Lin YJ, Wongcharoen W, Lo LW, Tuan TC, Udyavar AR, Chang SH, Tsao HM, Hsieh MH, Hu YF, Chen YJ, Chen SA. The efficacy of inducibility and circumferential ablation with pulmonary vein isolation in patients with paroxysmal atrial fibrillation. J Cardiovasc Electrophysiol 2007;18(6):607-11.

2. Oral H, Chugh A, Lemola K, Cheung P, Hall B, Good E, Han J, Tamirisa K, Bogun F, Pelosi F, Morady F. (2004). Noninducibility of atrial fibrillation as an end point of left atrial circumferential ablation for paroxysmal atrial fibrillation: A randomized study. Circulation 2004;110:2797-801.

3. Jaïs $P$, Hocini $M$, Sanders $P$, Hsu LF, Takahashi $Y$, Rotter M, Rostock T, Sacher F, Clementy J, Haissaguerre M. Long-term evaluation of atrial fibrillation ablation guided by noninducibility. Heart Rhythm 2006:3(2):140-5.

4. Fiala M, Bulková V, Škňouřil L, Nevřalová R, Toman O, Januška J, Špinar J, Wichterle D. Sinus rhythm restoration and arrhythmia noninducibility are major predictors of arrhythmia-free outcome after ablation for long-standing persistent atrial fibrillation: A prospective study. Heart Rhythm 2015;12(4):687-98.

5. Calkins H, Hindricks G, Cappato R, Kim Y, Saad E, Aguinaga L, Akar J, Badhwar V, Brugada J, Camm J, Chen P, Chen S, Chung M, Cosedis Nielsen J, Curtis A, Davies D, Day J, D'Avila A, Natasja De Groot N, Di Biase L, Duytschaever M, Edgerton J, Ellenbogen $K$, Ellinor $P$ Ernst S, Fenelon G, Gerstenfeld E, Haines D, Haissaguerre M, Helm R, Hylek E, Jackman W, Jalife J, Kalman J, Kautzner J, Kottkamp H, Kuck K, Kumagai K, Lee R, Lewalter T, Lindsay B, MacLe L, Mansour M, Marchlinski F, Michaud G, Nakagawa H, Natale A, Nattel S, Okumura K, Packer D, Pokushalov E, Reynolds M, Sanders P, Scanavacca M, Schilling R, Tondo C, Tsao H, Verma A, Wilber D, Yamane T. 2017 HRS/ EHRA/ECAS/APHRS/SOLAECE expert consensus statement on catheter and surgical ablation of atrial fibrillation: Executive summary. Europace 2018;20(1):157-208.
6. Costa FM, Ferreira AM, Oliveira S, Santos PG, Durazzo A, Carmo P, Santos KR, Cavaco D, Parreira L, Morgado F, Adragão P. Left atrial volume is more important than the type of atrial fibrillation in predicting the long-term success of catheter ablation. Int J Cardiol 2015;184:56-61.

7. Kumar S, Kalman JM, Sutherland F, Spence SJ, Finch S, Sparks PB Atrial fibrillation inducibility in the absence of structural heart disease or clinical atrial fibrillation: Critical dependence on induction protocol, inducibility definition, and number of inductions. Circ Arrhythm Electrophysiol 2012;5:531-6.

8. Vladinov G, Fermin L, Longini R, Ramos Y, Maratea E. Choosing the anesthetic and sedative drugs for supraventricular tachycardia ablations: A focused review. Pacing Clin Electrophysiol 2018;41(11):155563. doi: 10.1111/pace.13511

9. Mountantonakis SE, Elkassabany N, Kondapalli L, Marchlinski FE, Mandel JE, Hutchinson MD. Provocation of atrial fibrillation triggers during ablation: Does the use of general anesthesia affect inducibility? J Cardiovasc Electrophysiol 2015;26(1):16-20.

10. Fujii S, Zhou JR, Dhir A. Anesthesia for Cardiac Ablation. Cardiothorac Vasc Anesth 2018;32(4):1892-910. doi: 10.1053/j.jvca.2017.12.039

11. Richter B, Gwechenberger M, Filzmoser $P$, Marx M, Lercher $P$, Gössinger HD. Is inducibility of atrial fibrillation after radio frequency ablation really a relevant prognostic factor? Eur Heart J 2006;27(21):2553-9.

12. Shah S, Barakat AF, Saliba WI, Abdur Rehman K, Tarakji KG, Rickard J, Bassiouny M, Baranowski B, Tchou P, Bhargava M, Chung M, Dresing T, Callahan T, Cantillon D, Kanj M, Lindsay BD, Wazni OM, Hussein AA. Recurrent Atrial Fibrillation After Initial Long-Term Ablation Success: Electrophysiological Findings and Outcomes of Repeat Ablation Procedures. Circ Arrhythm Electrophysiol 2018;11(4):e005785.

13. Huang W, Liu T, Shehata M, Zhang K, Yao Y, Niu G, Amorn A, Liu X, Chugh SS, Wang $X$. Inducibility of atrial fibrillation in the absence of atrial fibrillation: what does it mean to be normal? Heart Rhythm 2011;8(4):489-92

14. Santangeli P, Zado ES, Garcia FC, Riley MP, Lin D, Frankel DS, Supple GE, Schaller RD, Dixit S, Callans DJ, Marchlinski FE. Lack of prognostic value of atrial arrhythmia inducibility and change in inducibility status after catheter ablation of atrial fibrillation. Heart Rhythm 2018;15(5):660-5.

15. Choi Jl, Pak HN, Park JS, Kwak JJ, Nagamoto Y, Lim HE, Park SW, Hwang $\mathrm{C}, \mathrm{Kim} \mathrm{YH}$. Clinical significance of early recurrences of atrial tachycardia after atrial fibrillation ablation. J Cardiovasc Electrophysiol 2010;21(12):1331-7. doi: 10.1111/j.1540-8167.2010.01831.x

16. Macle L, Khairy $P$, Weerasooriya R, Novak $P$, Verma A, Willems $S$, Arentz T, Deisenhofer I, Veenhuyzen G, Scavée C, Jaïs P, Puererfellner $H$, Levesque S, Andrade JG, Rivard L, Guerra PG, Dubuc M, Thibault B, Talajic M, Roy D, Nattel S, ADVICE trial investigators. Adenosineguided pulmonary vein isolation for the treatment of paroxysmal atrial fibrillation: an international, multicentre, randomised superiority trial. Lancet 2015;386(9994):672-9. 